\title{
Geodesic-Loxodromes for Diffusion Tensor Interpolation and Difference Measurement ${ }^{\star}$
}

\author{
Gordon Kindlmann ${ }^{1}$, Raúl San José Estépar ${ }^{1}$, Marc Niethammer², \\ Steven Haker ${ }^{1}$, and Carl-Fredrik Westin ${ }^{1}$ \\ ${ }^{1}$ Department of Radiology \\ ${ }^{2}$ Department of Psychiatry, Brigham and Women's Hospital, \\ Harvard Medical School, USA \\ gk@bwh.harvard .edu
}

\begin{abstract}
In algorithms for processing diffusion tensor images, two common ingredients are interpolating tensors, and measuring the distance between them. We propose a new class of interpolation paths for tensors, termed geodesic-loxodromes, which explicitly preserve clinically important tensor attributes, such as mean diffusivity or fractional anisotropy, while using basic differential geometry to interpolate tensor orientation. This contrasts with previous Riemannian and Log-Euclidean methods that preserve the determinant. Path integrals of tangents of geodesic-loxodromes generate novel measures of over-all difference between two tensors, and of difference in shape and in orientation.
\end{abstract}

\section{Introduction}

Diffusion tensor imaging (DTI) can uniquely discern the directional microstructure of living tissue 1. Certain mathematical attributes of diffusion tensors have established clinical value. The tensor trace is three times the bulk mean diffusivity (often referred to as apparent diffusion coefficient or ADC), and is used for rapid detection of ischemic stroke 2, and for detecting edema around brain lesions 3. Fractional anisotropy (FA) indicates the directional dependence of diffusion, and is currently the mainstay of DTI applications, because FA changes are associated with many neurological or psychiatric conditions 456].

Recent work has created a sophisticated mathematical context for diffusion tensors, considered as elements of a Riemannian manifold with a particular affine-invariant metric, derived from statistical or information-theoretic considerations $7 / 8$ 910 . The Log-Euclidean approach is a computationally efficient close approximation [1]. These methods are rigorous in that they explicitly respect the positive-definiteness of diffusivity. A consequence is that the determinant $\operatorname{det}(\mathbf{D})$ is given especial importance, since its level-set $\operatorname{det}(\mathbf{D})=0$ delimits the region of positive-definite tensors. Riemannian and Log-Euclidean tensor interpolation guarantee monotonic interpolation of the determinant.

\footnotetext{
* This work supported by NIH grants U41-RR019703, P41-RR13218, R01-MH050740, and R01-MH074794. DTI data courtesy of Dr. Susumu Mori, Johns Hopkins University, supported by NIH R01-AG20012-01 and P41-RR15241-01A1.
}

N. Ayache, S. Ourselin, A. Maeder (Eds.): MICCAI 2007, Part I, LNCS 4791, pp. 1-9, 2007.
(C) Springer-Verlag Berlin Heidelberg 2007 
We present a novel tensor interpolant, the geodesic-loxodrome, designed around clinically significant tensor properties. In navigation, loxodromes are paths of constant bearing. We generalize this to monotonically interpolate three tensor shape parameters, including tensor size and anisotropy. Geodesic-loxodromes are by definition the shortest path with the loxodrome properties, so they form geodesics on the manifold of tensors with fixed shape. In essence, geodesicloxodromes are minimal-length paths between tensors that monotonically interpolate tensor shape. By computing tangents to geodesic-loxodromes and projecting out different components of the tangent, we create novel shape-specific and orientation-specific measures of the large-scale difference between tensors.

For these tasks, we have found it advantageous to treat diffusion tensors as elements of a vector space. Linear transforms on $\mathbb{R}^{n}$ constitute a vector space isomorphic to $\mathbb{R}^{n \times n}[12$. Though often viewed as a covariance matrix, a diffusion tensor $\mathbf{D}$ is also a linear transform on $\mathbb{R}^{3}$ with the essential physical property of mapping (by Fick's first law) concentration gradient vector $\nabla c$ to diffusive flux vector $\mathbf{j}=-\mathbf{D} \nabla c[1]$. The set of positive-definite tensors is not a vector space (it is not closed under subtraction), but if one seeks merely to describe properties of given tensors, or to interpolate tensors in a convex manner, then the methods need not be explicitly designed around the positive-definiteness constraint.

\section{Theoretical Background}

Loxodromes are paths of constant bearing, or paths maintaining a fixed angle with north [13. Stated another way, let $\mathbf{p}(\theta, \phi)=(r \cos (\theta) \sin (\phi)$, $r \sin (\theta) \sin (\phi), r \cos (\phi))$ be a parameterization of a radius- $r$ globe in $\mathbb{R}^{3}$ with $\mathbf{p}(0,0)$ at the north pole. Then $\mathbf{n}(\mathbf{x})=-\partial \mathbf{p} /\left.\partial \phi\right|_{\mathbf{p}^{-1}(\mathbf{x})}$ is a tangent to the sphere, pointing north. Let $\widehat{\mathbf{n}}(\mathbf{x})=\mathbf{n}(\mathbf{x}) /|\mathbf{n}(\mathbf{x})|$. Then, a loxodrome with unit speed and bearing $\cos ^{-1}(\alpha)$ is traced by a path $\gamma(t)$ on the globe for which:

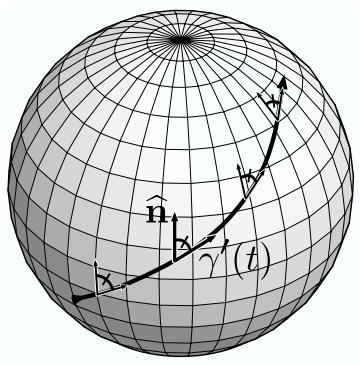

$$
\left|\gamma^{\prime}(t)\right|=1 \text { and } \gamma^{\prime}(t) \cdot \widehat{\mathbf{n}}(\gamma(t))=\alpha \text { for all } t .
$$

The path tangent $\gamma^{\prime}(t)$ is also tangent to the sphere, and its constant inner product with $\widehat{\mathbf{n}}$ implies that $\gamma(t)$ moves northward (or southward) at a constant rate. Our geodesic-loxodromes similarly move along certain tensor shape parameters at a constant rate, thereby monotonically interpolating tensor shape. We now review the mathematics needed to define geodesic-loxodromes, including the theoretical distinction between tensor shape and orientation.

Notation. The six-dimensional vector space of second-order symmetric tensors is notated $\mathrm{Sym}_{3}$. Tensor contraction $\mathbf{A}: \mathbf{B}=\operatorname{tr}\left(\mathbf{A B}^{\top}\right)$ is an inner product on $\mathrm{Sym}_{3}$, and the norm is $|\mathbf{D}|=\sqrt{\mathbf{D}: \mathbf{D}}$. The tensor $\mathbf{A}$ can be decomposed into isotropic and deviatoric parts, defined by $\overline{\mathbf{A}}=\operatorname{tr}(\mathbf{A}) \mathbf{I} / 3$ and $\widetilde{\mathbf{A}}=\mathbf{A}-\overline{\mathbf{A}}$, respectively. $\mathbf{I}$ is the identity tensor. $\mathrm{SO}_{3}$ is the group of rotations on $\mathbb{R}^{3}$. The group action $\psi: \mathrm{SO}_{3} \times \mathrm{Sym}_{3} \mapsto \mathrm{Sym}_{3}, \psi(\mathbf{R}, \mathbf{D})=\mathbf{R D R} \mathbf{R}^{\top}$ defines a mapping on 
$\mathrm{Sym}_{3}$ for each rotation $\mathbf{R}$ in $\mathrm{SO}_{3}$. The orbit of $\mathbf{D}$ is $\mathrm{SO}_{3}(\mathbf{D})=\left\{\mathbf{R D R} \mathbf{R}^{\top} \mid \mathbf{R} \in\right.$ $\left.\mathrm{SO}_{3}\right\}$; informally it is all possible rotations of $\mathbf{D}$. The orbits of $\psi$ partition $\mathrm{Sym}_{3}$ into equivalence classes because $\mathrm{SO}_{3}$ is a group. We can then say that tensors $\mathbf{A}$ and $\mathbf{B}$ have the same shape if they are on the same orbit $\mathrm{SO}_{3}(\mathbf{A})=\mathrm{SO}_{3}(\mathbf{B})$, which is equivalent to saying they have the same three eigenvalues. A tensor invariant $J: \mathrm{Sym}_{3} \mapsto \mathbb{R}$ can be defined as a scalar-valued function of tensors that is constant on orbits: $\mathrm{SO}_{3}\left(\mathbf{D}_{0}\right)=\mathrm{SO}_{3}\left(\mathbf{D}_{1}\right) \Rightarrow J\left(\mathbf{D}_{0}\right)=J\left(\mathbf{D}_{1}\right)$. Trace $\operatorname{tr}()$ and determinant $\operatorname{det}()$ are invariants, as are the tensor eigenvalues.

Just as scalar-valued functions over the vector space $\mathbb{R}^{3}$ have gradients with values in $\mathbb{R}^{3}$, tensor invariants have gradients with values in $\mathrm{Sym}_{3} \sqrt{1}$. Adapting the $\nabla$ notation from vector calculus, we use $\nabla J(\mathbf{D})$ to represent the tensor-valued gradient of invariant $J$, evaluated at tensor D. $\nabla J$ "points" in the direction along which $J$ increases fastest. Deriving expressions for gradients of standard invariants is in continuum mechanics texts [14], for example $\boldsymbol{\nabla} \operatorname{tr}(\mathbf{D})=\mathbf{I}$ and $\boldsymbol{\nabla} \operatorname{det}(\mathbf{D})=\operatorname{det}(\mathbf{D}) \mathbf{D}^{-1}$. Any invariant $J$ is constant on orbits of $\psi$, thus $\boldsymbol{\nabla} J(\mathbf{D})$ is orthogonal at $\mathbf{D}$ to the orbit $\mathrm{SO}_{3}(\mathbf{D})$. We can then say that near $\mathbf{D}, \boldsymbol{\nabla} J(\mathbf{D})$ spans one degree of freedom in tensor shape. For tensors in $\mathrm{Sym}_{3}$, shape has three degrees of freedom (because the three eigenvalues are independent), and we intend to define at each tensor $\mathbf{D}$ an orthonormal basis for shape variation.

Orthogonal Invariants. We build upon work by Ennis and Kindlmann that advocates sets of orthogonal invariants for DTI analysis [15]. Invariants $J_{1}$ and $J_{2}$ are said to be orthogonal if $\boldsymbol{\nabla} J_{1}(\mathbf{D}): \nabla J_{2}(\mathbf{D})=0$ for all $\mathbf{D}$. We adopt the same two sets of orthogonal invariants $\left\{K_{i}\right\}$ and $\left\{R_{i}\right\}$ as in [15], because they contain trace and FA, which we intend to preserve in our method:

$$
\begin{array}{ll}
K_{1}(\mathbf{D})=\operatorname{tr}(\mathbf{D}) & R_{1}(\mathbf{D})=|\mathbf{D}| \\
K_{2}(\mathbf{D})=|\widetilde{\mathbf{D}}| & R_{2}(\mathbf{D})=\mathrm{FA}(\mathbf{D})=\sqrt{\frac{3 \widetilde{\mathbf{D}}: \widetilde{\mathbf{D}}}{2 \mathbf{D}: \mathbf{D}}} \\
K_{3}(\mathbf{D})=\operatorname{mode}(\mathbf{D}) & R_{3}(\mathbf{D})=K_{3}(\mathbf{D})=\operatorname{mode}(\mathbf{D})
\end{array}
$$

where mode $(\mathbf{D})=3 \sqrt{6} \operatorname{det}(\widetilde{\mathbf{D}} /|\widetilde{\mathbf{D}}|)$. Both sets include measures of size (either trace $K_{1}$ or norm $R_{1}$ ) and of anisotropy (either eigenvalue standard deviation $K_{2}$ or fractional anisotropy $\left.R_{2}\right)$, and both use mode $\left(K_{3}=R_{3}\right)$ to distinguish linear $(\operatorname{mode}=+1)$ from planar $(\operatorname{mode}=-1)$ anisotropy. Trace $\left(K_{1}\right)$ and FA $\left(R_{2}\right)$ are not orthogonal, and so are in different invariant sets [15]. The determinant is another measure of size, but we are unaware of two complementary invariants that, with determinant, constitute an orthogonal invariant set. We restate from [15] the formulae for the tensor-valued gradients of $K_{i}$ and $R_{i}$ :

$$
\begin{array}{rlrl}
\nabla K_{1}(\mathbf{D}) & =\mathbf{I} & \nabla R_{1}(\mathbf{D})=\mathbf{D} /|\mathbf{D}| \\
\nabla K_{2}(\mathbf{D})=\boldsymbol{\Theta}(\mathbf{D})=\widetilde{\mathbf{D}} /|\widetilde{\mathbf{D}}| & \nabla R_{2}(\mathbf{D})=\sqrt{\frac{3}{2}}\left(\frac{\mathbf{\Theta}(\mathbf{D})}{|\mathbf{D}|}-\frac{|\widetilde{\mathbf{D}}| \mathbf{D}}{|\mathbf{D}|^{3}}\right) \\
\nabla K_{3}(\mathbf{D})=\frac{3 \sqrt{6} \mathbf{\Theta}(\mathbf{D})^{2}-3 K_{3}(\mathbf{D}) \Theta(\mathbf{D})-\sqrt{6} \mathbf{I}}{K_{2}(\mathbf{D})} & \nabla R_{3}(\mathbf{D})=\nabla K_{3}(\mathbf{D}) .
\end{array}
$$

\footnotetext{
${ }^{1}$ We omit the distinction between covariant and contravariant vectors as we use only
} orthonormal bases, whose orthonormality is preserved by our group action $\psi$ [14. 
We notate the normalized gradient of invariant $J$ as $\widehat{\nabla} J(\mathbf{D})=\nabla J(\mathbf{D}) /|\boldsymbol{\nabla} J(\mathbf{D})|$. Thus, at each tensor D, $\left\{\widehat{\nabla} K_{i}(\mathbf{D})\right\}$ and $\left\{\widehat{\nabla} R_{i}(\mathbf{D})\right\}$ form orthonormal bases for local shape variation: nearby tensors along those directions differ only in shape, not orientation, from D. Note that tensor diagonalization is not required to compute either the invariants or their gradients.

\section{Methods}

Interpolation. We define the geodesic-loxodrome $\gamma(t)$ between $\mathbf{A}$ and $\mathbf{B}$ in $\mathrm{Sym}_{3}$ as the shortest path satisfying (compare to Equation 1):

$$
\begin{aligned}
& \gamma(0)=\mathbf{A}, \quad \gamma(l)=\mathbf{B}, \quad\left|\gamma^{\prime}(t)\right|=1, \text { and } \\
& \gamma^{\prime}(t): \widehat{\nabla} J_{i}(\gamma(t))=\alpha_{i} \text { for all } t \in[0, l], i \in\{1,2,3\}
\end{aligned}
$$

where $l$ and $\alpha_{i}$ are constants that characterize the path, and either $J_{i}=K_{i}$ or $J_{i}=R_{i}$. By using normalized invariant gradients $\left(\widehat{\nabla} J_{i}\right.$ instead of $\left.\nabla J_{i}\right), \gamma(t)$ depends on the tensor shape degree of freedom parameterized by $J_{i}$, but not on the parameterization rate $\left|\nabla J_{i}\right|$, which in general is not constant. $\gamma(t)$ linearly interpolates invariants with constant-magnitude gradients (trace $K_{1}, K_{2}$, and norm $R_{1}$ ); other invariants are merely monotonically interpolated:

$$
\frac{d}{d t} J_{i}(\gamma(t))=\gamma^{\prime}(t): \nabla J_{i}(\gamma(t))=\gamma^{\prime}(t): \widehat{\nabla} J_{i}(\gamma(t))\left|\nabla J_{i}(\gamma(t))\right|=\alpha_{i}\left|\nabla J_{i}(\gamma(t))\right| .
$$

$\alpha_{i}$ is constant and $\left|\nabla J_{i}(\gamma(t))\right| \geq 0$, thus $\frac{d}{d t} J_{i}(\gamma(t))$ has fixed sign, and $J_{i}(\gamma(t))$ is monotonic. In particular, if $J_{i}(\mathbf{A})=J_{i}(\mathbf{B})$ for some $i, J_{i}(\gamma(t))$ is constant, and $\gamma(t)$ lies in a level-set $\mathcal{L}_{i}$ of $J_{i}$. If $J_{i}(\mathbf{A})=J_{i}(\mathbf{B})$ for all $i=1,2,3$, then $\gamma(t)$ lies within the intersection $\cap_{i} \mathcal{L}_{i}$. However, $\cap_{i} \mathcal{L}_{i}$ is exactly the orbit $\mathcal{O}=$ $\mathrm{SO}_{3}(\mathbf{A})=\mathrm{SO}_{3}(\mathbf{B})$ of $\psi$, because the three $J_{i}$ determine the $\lambda_{i}$, and tensors with equal eigenvalues are on the same orbit. As $\gamma(t)$ is by definition the shortest such path in $\mathcal{O}$, by the Hopf-Rinow-de Rham theorem it is a geodesic on $\mathcal{O}[16$. Space does not permit a proof, but conditions for the theorem are met because $\mathcal{O}$ is a closed subset of a complete metric space. We also note without proof that geodesics on $\mathcal{O}$ are not simply images under $\psi$ of geodesics on $\mathrm{SO}_{3}$, because the extrinsic curvatures of orbits are non-uniformly scaled by eigenvalue differences.

Distance Measurement. A distance $d(\mathbf{A}, \mathbf{B})$ between $\mathbf{A}$ and $\mathbf{B}$ can be defined in terms of the geodesic-loxodrome $\gamma(t)$ connecting them: $d(\mathbf{A}, \mathbf{B})=$ $\int_{0}^{l}\left|\gamma^{\prime}(t)\right| d t=l$. However, the normalized invariant gradients $\widehat{\nabla} J_{i}$ can also decompose the path tangent $\gamma^{\prime}(t)$ into mutually orthogonal shape $\boldsymbol{\sigma}(t)$ and orientation $\boldsymbol{\omega}(t)$ tangents. From these we define shape $d_{s h}$ and orientation $d_{\text {or }}$ distances, which measure large-scale differences specifically in shape and orientation:

$$
\begin{aligned}
\boldsymbol{\sigma}(t)=\sum_{i} \boldsymbol{\gamma}^{\prime}(t): \widehat{\nabla} J_{i}(\boldsymbol{\gamma}(t)) \widehat{\nabla} J_{i}(\boldsymbol{\gamma}(t)) & \rightarrow d_{s h}(\mathbf{A}, \mathbf{B})=\int_{0}^{l}|\boldsymbol{\sigma}(t)| d t \\
\boldsymbol{\omega}(t)=\gamma^{\prime}(t)-\boldsymbol{\sigma}(t) & \rightarrow d_{o r}(\mathbf{A}, \mathbf{B})=\int_{0}^{l}|\boldsymbol{\omega}(t)| d t
\end{aligned}
$$

Implementation. Our initial investigation of geodesic-loxodromes has focused on their theoretical definition and properties, rather than on a fast numerical 
solution. We describe here a brute-force gradient-descent scheme for updating vertices of a discretized polyline through $\mathrm{Sym}_{3}$ (initialized with linear interpolation) so that it converges to the geodesic-loxodrome. For any vertex $\mathbf{D}_{n}$, let $\mathbf{D}^{-}=\left(\mathbf{D}_{n}+\mathbf{D}_{n-1}\right) / 2$ and $\mathbf{D}^{+}=\left(\mathbf{D}_{n+1}+\mathbf{D}_{n}\right) / 2$ be the (linearly) interpolated tensor values at the edge midpoints around $\mathbf{D}_{n}$, and let $\mathbf{T}^{-}=\mathbf{D}_{n}-\mathbf{D}_{n-1}$ and $\mathbf{T}^{+}=\mathbf{D}_{n+1}-\mathbf{D}_{n}$ be the (non-normalized) tangents into, and away from, $\mathbf{D}_{n}$.

Then, $r_{i}\left(\mathbf{D}_{n}\right)=\mathbf{T}^{+}: \widehat{\nabla} J_{i}\left(\mathbf{D}^{+}\right)-\mathbf{T}^{-}: \widehat{\nabla} J_{i}\left(\mathbf{D}^{-}\right)$is the change in the projection of the path tangent onto $\widehat{\nabla} J_{i}$, which is zero on a loxodrome, and $\kappa\left(\mathbf{D}_{n}\right)=\mid \mathbf{T}^{+}-$ $\mathbf{T}^{-} \mid$is approximately proportional to the curvature at $\mathbf{D}_{n}$, which is minimized on a minimal-length path. Gradient descent on $r_{i}^{2}$ and $\kappa^{2}$ leads to the update rule $\mathbf{D}_{n} \leftarrow \mathbf{D}_{n}+\delta\left(\sum_{i} r_{i}\left(\mathbf{D}_{n}\right) \hat{\nabla} J_{i}\left(\mathbf{D}_{n}\right)+\mathbf{U}-\sum_{i}\left(\mathbf{U}: \hat{\nabla} J_{i}\left(\mathbf{D}_{n}\right)\right) \hat{\nabla} J_{i}\left(\mathbf{D}_{n}\right)\right)$, where $\mathbf{U}=\mathbf{D}_{n-1}-2 \mathbf{D}_{n}+\mathbf{D}_{n+1}$, and $\delta$ is the time increment. Note that the curveshortening curvature flow along $\mathbf{U}$ is only allowed to act along the subspace (of orientation change) orthogonal to the $\widehat{\nabla} J_{i}\left(\mathbf{D}_{n}\right)$. After all vertices have been so updated, vertices are moved along the polyline to enforce equi-distant spacing (constant-rate parameterization). Results here use a 100-point polyline with $\delta=$ 0.1 , converging sufficiently in about one second on a modern PC. Distances (Eq. 5) are computed by summing (over the polyline) the lengths of the segments (for $d(\mathbf{A}, \mathbf{B})$ ), or their projections onto the span of the local $\widehat{\nabla} J_{i}\left(\right.$ for $\left.d_{s h}(\mathbf{A}, \mathbf{B})\right)$, or the complements of these projections (for $d_{\text {or }}(\mathbf{A}, \mathbf{B})$ ).

\section{Results}

We demonstrate geodesic-loxodromes with glyphs and with plots of invariants along interpolation paths. Tensors are shown as superquadric glyphs [17]. For qualitative comparisons, the invariant plots (on the right side of the figures) are individually scaled (mode $K_{3}$ is also shifted). Figure 1 interpolates two linearly anisotropic tensors of different size and orientation. By their definitions, the first three methods monotonically interpolate one invariant: the tensor trace $\left(K_{1}\right)$ with linear interpolation (Fig. 1(a) , and the tensor determinant with Riemannian [78910] (Fig. 1(b) and Log-Euclidean 11] (Fig. 1(c) interpolation. By monotonically interpolating three orthogonal invariants, however, geodesicloxodromes fully control tensor shape. In particular, the cylindrical shape is maintained in Fig. 1(d) by fixing the anisotropy type (tensor mode $K_{3}$ ).

Figure 2 interpolates two tensors with equal size (as measured by norm $R_{1}=$ $|\mathbf{D}|)$, but unequal FA $\left(R_{2}\right)$, mode $\left(R_{3}\right)$, and orientation. As in Fig. 1, Riemannian results were very similar to Log-Euclidean, and so are not shown. Log-Euclidean interpolation (Fig. 2(a) does not monotonically interpolate norm $\left(R_{1}\right)$ or FA $\left(R_{2}\right)$, and the wide range of mode $\left(R_{3}\right)$ leads to planar anisotropy dissimilar to the endpoints. The geodesic-loxodrome (Fig. 2(b) , on the other hand, maintains the norm, and monotonically interpolates the other invariants. Though not part of the geodesic-loxodrome formulation, in this case the tensor determinant is also monotonically interpolated; the apparent lengthening is due to rotation.

Figure 3 demonstrates distance measures on a single slice of a DTI scan (pixel size $1.5^{3} \mathrm{~mm}$ ). The cerebral spinal fluid (CSF) is bright in the tensor trace 


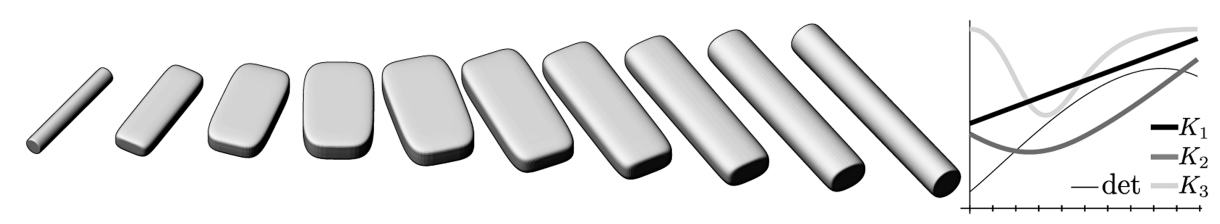

(a) Linear interpolation: $(1-t) \mathbf{A}+t \mathbf{B}$

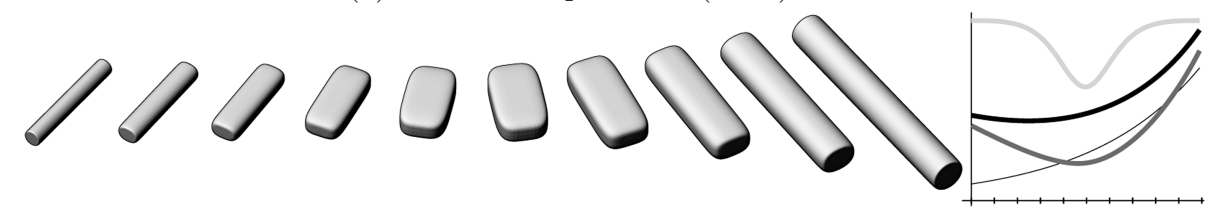

(b) Riemannian interpolation: $\mathbf{A}^{1 / 2}\left(\mathbf{A}^{-1 / 2} \mathbf{B} \mathbf{A}^{-1 / 2}\right)^{t} \mathbf{A}^{1 / 2}$

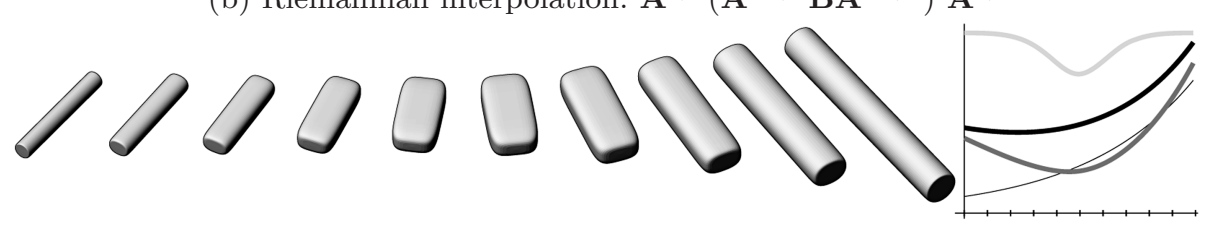

(c) Log-Euclidean interpolation: $\exp ((1-t) \log (\mathbf{A})+t \log (\mathbf{B}))$

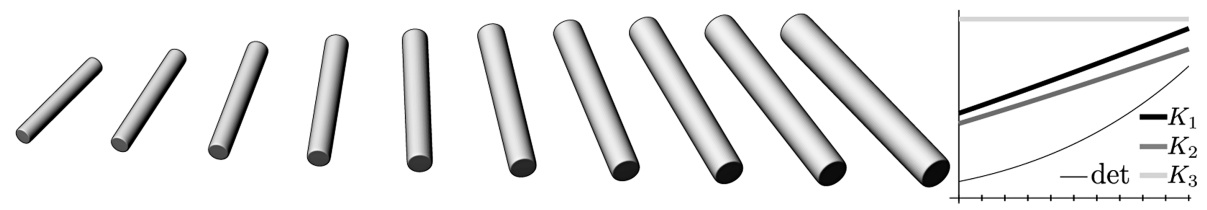

(d) Geodesic-loxodrome interpolation based on $K_{i}$ invariants

Fig. 1. Interpolations between two cylindrical tensors. Trace $\left(K_{1}\right)$ is linearly interpolated in (a), and determinant is monotonically interpolated in (b) and (c). Geodesicloxodromes (d) monotonically interpolate all $K_{i}$ as well as (in this case) the determinant. Constancy of mode $\left(K_{3}\right)$ wholly avoids planar anisotropy at intermediate values.

image (Fig. 3(a) , and the white matter is bright in the FA image (Fig. 3(b)], both of which show a square centered on a reference pixel in the corpus callosum splenium (left-right oriented linear anisotropy). Subsequent subfigures show measured distances between the tensors at each pixel and at the reference pixel. The linear (Fig. 3(c) ), Log-Euclidean (Fig. 3(d)), and geodesic-loxodrome $d$ distances (Fig. 3(e) differ in brightness and contrast but are otherwise qualitatively similar. However, the geodesic-loxodrome shape distance $d_{s h}$ (Fig. 3(f) and orientation distance $d_{\text {or }}$ (Fig. $3(\mathrm{~g})$ highlight different structures. The shape distance $d_{s h}$ is consistently low throughout the white matter, since these voxels all have similar shape. The orientation distance $d_{\text {or }}$, on the other hand, is low in the gray matter, since there is little orientation change between isotropic and anisotropic tensors of comparable size. Within the white matter (where FA is highest), $d_{\text {or }}$ successfully distinguishes between left-right and other orientations (where $d_{\text {or }}$ is low and high, respectively). These results suggest that the novel shape and 

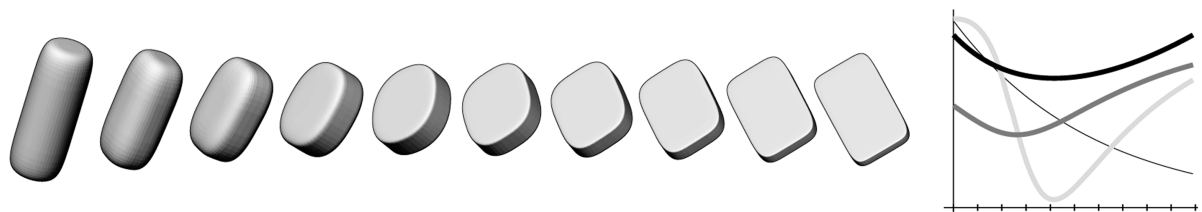

(a) Log-Euclidean interpolation
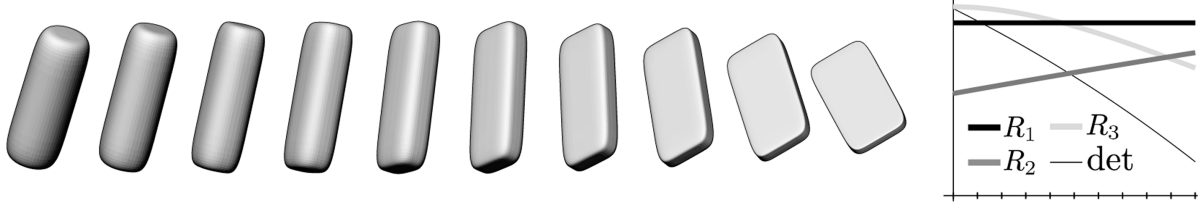

(b) Geodesic-loxodrome interpolation based on $R_{i}$ invariants

Fig. 2. Log-Euclidean and geodesic-loxodrome interpolation between two tensors with equal $R_{1}$ (tensor norm). By monotonically interpolating FA $\left(R_{2}\right)$ and anisotropy type (mode $R_{3}$ ), geodesic-loxodromes better maintain tensor shape during rotation.

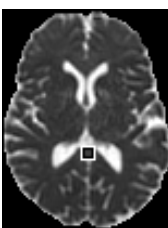

(a) $\operatorname{tr}$

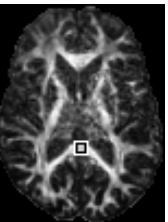

(b) FA

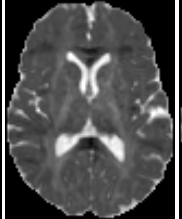

(c) Lin.

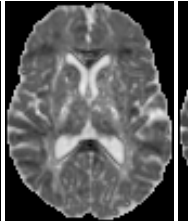

(d) L-E

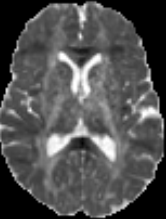

(e) G-L $d$

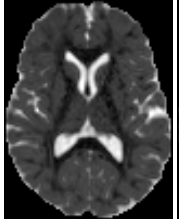

(f) G-L $d_{s h}$

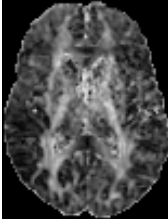

(g) G-L $d_{o r}$

Fig. 3. DTI slice with distance measures. The tensor trace (a) and FA (b) images indicate (small square) the reference white-matter voxel to which distances are measured. Note that the geodesic-loxodrome shape distance $d_{s h}$ (f) is low inside white matter.

orientation distance measures $\left(d_{s h}\right.$ and $\left.d_{o r}\right)$ based on geodesic-loxodromes can better differentiate neuroanatomic structures than previous distance measures, highlighting their possible utility for segmentation.

\section{Discussion and Future Work}

This work was inspired by how the Riemannian and Log-Euclidean methods guarantee monotonic interpolation of a particular invariant, the determinant. Geodesic-loxodromes monotonically interpolate not one but three tensor invariants, which by their orthogonality completely determine tensor shape, and which by design include at least one clinically significant invariant, either trace $\left(K_{1}\right)$ or FA $\left(R_{2}\right)$. Choosing between $J_{i}=K_{i}$ or $J_{i}=R_{i}$ depends on desired path properties: the $K_{i}$ are better if mean diffusivity $\left(K_{1} / 3\right)$ is an essential tissue parameter for the particular application, while $R_{i}$ may be better if FA $\left(R_{2}\right)$ values are fundamental, although the actual differences between using $K_{i}$ and $R_{i}$ are small. Our results highlight the importance of controlling anisotropy type 
(tensor mode) during interpolation, which is unaddressed by previous methods. Geodesic-loxodromes demonstrate the mapping of an intuitive distinction between shape and orientation into a mathematical formulation of interpolation and distance measurement. In this light, the most closely related previous work is by Chefd'hotel et al., in which images of diffusion tensors are regularized by an "isospectral" flow that smoothes orientations but maintains shape [18].

We are uncertain whether geodesic-loxodromes are geodesics on some sixdimensional Riemannian manifold embedded in $\mathrm{Sym}_{3}$. However, we believe it should be possible to generalize to geodesic-loxodromes the notion of a weighted intrinsic mean of multiple tensors on a Riemannian manifold 8910, which would lead to a novel method of interpolating three-dimensional images of tensor samples. This requires fast numerical methods for computing geodesic-loxodromes and their exponential map counterparts (finding the geodesic-loxodrome given its starting point and initial tangent), which is our current focus. We are also studying how to apply our distance measures to segmentation, including optimizing a weighted combination of $d_{s h}$ and $d_{\text {or }}$ into a single distance measure.

\section{References}

1. Basser, P.J., Mattiello, J., Bihan, D.L.: Estimation of the effective self-diffusion tensor from the NMR spin-echo. J. Mag. Res. B B103(3), 247-254 (1994)

2. Sotak, C.H.: The role of diffusion tensor imaging in the evaluation of ischemic brain injury - A review. NMR in Biomedicine 15, 561-569 (2002)

3. Lu, S., Ahn, D., Johnson, G., Law, M., Zagzag, D., Grossman, R.I.: Diffusiontensor MR imaging of intracranial neoplasia and associated peritumoral edema: Introduction of the tumor infiltration index. Neurorad. 232(1), 221-228 (2004)

4. Thomalla, G., Glauche, V., Koch, M A, Beaulieu, C., Weiller, C., Röther, J.: Diffusion tensor imaging detects early Wallerian degeneration of the pyramidal tract after ischemic stroke. NeuroImage 22(4), 1767-1774 (2004)

5. Salat, D.H., Tuch, D.S., Hevelone, N.D., Fischl, B., Corkin, S., Rosas, H.D., Dale, A.M.: Age-related changes in prefrontal white matter measured by diffusion tensor imaging. Annals of the New York Academy of Sciences 1064, 37-49 (2005)

6. Tuch, D.S., Salat, D.H., Wisco, J.J., Zaleta, A.K., Hevelone, N.D., Rosas, H.D.: Choice reaction time performance correlates with diffusion anisotropy in white matter pathways supporting visuospatial attention. Proc. Nat. Acad. Sci. 102(34), 12212-12217 (2005)

7. Batchelor, P.G., Moakher, M., Atkinson, D., Calamante, F., Connelly, A.: A rigorous framework for diffusion tensor calculus. Mag. Res. Med. 53(1), 221-225 (2005)

8. Fletcher, P.T., Joshi, S.: Riemannian geometry for the statistical analysis of diffusion tensor data. Signal Processing 87(2), 250-262 (2007)

9. Lenglet, C., Rousson, M., Deriche, R., Faugeras, O.: Statistics on the manifold of multivariate normal distributions: Theory and application to diffusion tensor MRI processing. J. Math. Imaging Vis. 25(3), 423-444 (2006)

10. Pennec, X., Fillard, P., Ayache, N.: A Riemannian framework for tensor computing. Int. J. Comp. Vis. 66(1), 41-66 (2006)

11. Arsigny, V., Fillard, P., Pennec, X., Ayache, N.: Log-Euclidean metrics for fast and simple calculus on diffusion tensors. Mag. Res. Med. 56(2), 411-421 (2006) 
12. Hoffman, K., Kunze, R.: Linear Algebra, ch. 3,8. Prentice-Hall, Inc. Englewood Cliffs, NJ (1971)

13. Pearson, F.: Map Projection: Theory and Applications, ch. 8. CRC Press, Boca Raton, FL (1990)

14. Holzapfel, G.A.: Nonlinear Solid Mechanics. John Wiley and Sons, Ltd. England (2000)

15. Ennis, D.B., Kindlmann, G.: Orthogonal tensor invariants and the analysis of diffusion tensor magnetic resonance images. Mag. Res. Med. 55(1), 136-146 (2006)

16. Petersen, P.: Riemannian Geometry. Springer, Heidelberg (1997)

17. Ennis, D.B., Kindlman, G., Rodriguez, I., Helm, P.A., McVeigh, E.R.: Visualization of tensor fields using superquadric glyphs. Mag. Res. Med. 53, 169-176 (2005)

18. Chefd'hotel, C., Tschumperlé, D., Deriche, R., Faugeras, O.: Regularizing flows for constrained matrix-valued images. J. Math. Imaging Vis. 20(1-2), 147-162 (2004) 